\section{Impact Factor Dynamics}

\author{
Peter C. Black, Editor-in-Chief ${ }^{\bowtie}$
}

Soc Int Urol J.2021;2(5):271-272

DOI: 10.48083/ZKHI7989

Every journal has its particular focus and also mission. The SIUJ, for example, strives for global representation and to provide relevant content to the global urologic audience. However, all journals share the goal of disseminating high-quality scientific content and, to some degree, journals are competing with one another for the same content. That is where the impact factor (IF) comes in.

For better or worse, the IF has become the principle quantitative measure of quality of a journal. This is one of several factors that authors use to select a journal to which to submit their scientific articles. Another factor may be the target audience of the journal. A paper may be better suited for a subspeciality journal rather than one targeting a broader audience. Journals in fields of research with a smaller audience may have lower IFs but still maintain high quality. A journal may develop strategies to maximize IF by developing content known to attract many citations (eg, review papers and guidelines), while another journal in the same field choses instead to provide a platform for publication across a broader spectrum of the specialty. The quality of the content in these two journals may be the same, but the former will claim a higher IF and the latter will feel the pressure to change publication policies in an attempt to match the higher impact journal.

Journals with higher IFs attract higher quality manuscripts, which makes the process self-fulfilling. On the other hand, new journals like the SIUJ can struggle to attract articles because of the lack of IF, which takes several years to obtain. Indeed, many prospective authors question why they should submit to journals like the SIUJ if they cannot list an IF in their curriculum vitae. The only motivation is for authors to support the mission and philosophy of the journal with the intent of building a high-quality journal that will eventually have a desirable IF. The European Urology family of journals has done a remarkable job in this regard.

We have observed IF inflation in all areas of medical publishing, including urologic publishing. There are numerous new urologic journals available. This implies that more papers are being published, which would mean that there is more opportunity for published papers to be cited. On the other hand, this also means that citations could be spread over more papers, which would result in IF deflation. In an analysis by Althouse et al. in 2008, most of the increase in IF was attributed to more references per manuscript rather than more manuscripts[1]. The weighted IF for 23 urologic journals in 2004 was 2.132 , and it increased by $3.2 \%$ annually between 1995 and 2004. In their analysis, however, just as many journals left the field as entered the field, and that is not the case in urologic publishing in recent years. They reported that larger fields do not have larger IFs based on size alone. However, if every paper cites key articles on the topic of investigation, and there are many more papers, it does stand to reason that some journals will see an increase in IF due to this increase in publication.

The digital boom and open access could also contribute to IF inflation. Easier access to journal articles allows easier citation. A trip to the library and coins for the photocopy machine are no longer required to read a published article. In this regard, IF inflation is a positive trend. It is also positive if it means more investigators are conducting and publishing research.

There is no question that there are significant flaws in the use of the IF as a measure of quality research and especially as a measure of an individual researcher's output, which can be used to determine funding and promotion. The IF of a journal is ultimately determined by technical factors not related to an article's quality[2]. Utrecht University in the Netherlands has recently made a very clear statement with respect to the simmering debate about use of IF in academia[3]. This institution will abandon the use of the IF in all hiring and promotion decisions. Instead, they will judge scholars on the basis of commitment to teamwork, efforts to promote open science, and other measures. Means to evaluate those measures need to be established. Other institutions will likely follow the lead of Utrecht University, but there is no reason yet to think that the importance of the IF in publishing and in academia will decline any time soon. 


\section{References}

1. Althouse, BM West JD, Bergstrom T, Bergstrom CT. Differences in impact factor across fields and over time. 2008 April 23. Available at: https://escholarship.org/uc/item/76h442pg. Accessed August 7, 2021.

2. Seglen P0. Why the impact factor of journals should not be used for evaluating research. BMJ.1997Feb 15;314(7079):498-502. doi: 10.1136/bmj.314.7079.497
3. Woolston C. Impact factor abandoned by Dutch university in hiring and promotion decisions. Nature.2021 June 25;595:462. Available at: https://www.nature.com/articles/d41586-021-01759-5. Accessed August 7, 2021. doi: https://doi.org/10.1038/d41586-021-01759-5 